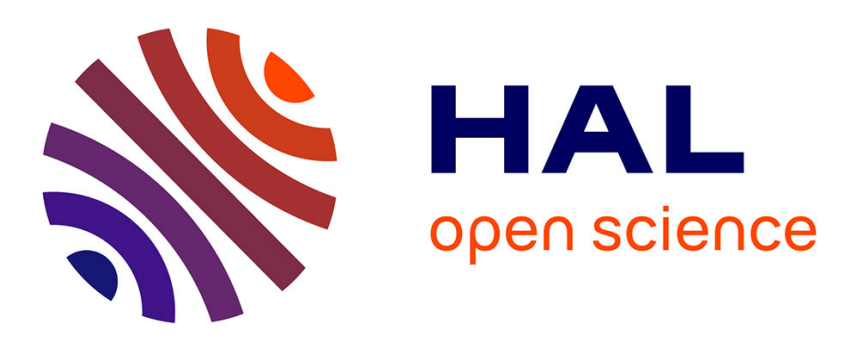

\title{
Diffusion MRI Signal Reconstruction with Continuity Constraint and Optimal Regularization
}

\author{
Emmanuel Caruyer, Rachid Deriche
}

\section{To cite this version:}

Emmanuel Caruyer, Rachid Deriche. Diffusion MRI Signal Reconstruction with Continuity Constraint and Optimal Regularization. Medical Image Analysis, 2012, 16 (6), pp.1113-1120. 10.1016/j.media.2012.06.011 . hal-00711883

\section{HAL Id: hal-00711883 \\ https://hal.inria.fr/hal-00711883}

Submitted on 26 Jun 2012

HAL is a multi-disciplinary open access archive for the deposit and dissemination of scientific research documents, whether they are published or not. The documents may come from teaching and research institutions in France or abroad, or from public or private research centers.
L'archive ouverte pluridisciplinaire HAL, est destinée au dépôt et à la diffusion de documents scientifiques de niveau recherche, publiés ou non, émanant des établissements d'enseignement et de recherche français ou étrangers, des laboratoires publics ou privés. 


\title{
Diffusion MRI Signal Reconstruction with Continuity Constraint and Optimal Regularization
}

\author{
Emmanuel Caruyer ${ }^{\mathrm{a},}$, Rachid Deriche ${ }^{\mathrm{a}}$ \\ ${ }^{a}$ Athena Project-Team, Inria, 2004 Route des Lucioles, BP93, 06902 Sophia-Antipolis Cedex, France.
}

\begin{abstract}
In diffusion MRI, the reconstruction of the full Ensemble Average Propagator (EAP) provides new insights in the diffusion process and the underlying microstructure. The reconstruction of the signal in the whole Q-space is still extremely challenging however. It requires very long acquisition protocols, and robust reconstruction to cope with the very low SNR at large $b$-values. Several reconstruction methods were proposed recently, among which the Spherical Polar Fourier (SPF) expansion, a promising basis for signal reconstruction. Yet the reconstruction in SPF is still subject to noise and discontinuity of the reconstruction. In this work, we present a method for the reconstruction of the diffusion attenuation in the whole Q-space, with a special focus on continuity and optimal regularization. We derive a modified Spherical Polar Fourier (mSPF) basis, orthonormal and compatible with SPF, for the reconstruction of a signal with continuity constraint. We also derive the expression of a Laplace regularization operator in the basis, together with a method based on generalized cross validation for the optimal choice of the parameter. Our method results in a noticeable dimension reduction as compared with SPF. Tested on synthetic and real data, the reconstruction with this method is more robust to noise and better preserves fiber directions and crossings.
\end{abstract}

Keywords: Diffusion MRI, Laplace Regularization, Q-space imaging,

\section{Introduction}

In diffusion MRI, the acquisition and reconstruction of the signal attenuation on the 3D Q-space allows reconstruction of the full probability of water molecules displacement, known as the ensemble average propagator (EAP). The radial and angular information contained in the EAP opens a wide range of applications, such as the definition of new biomarkers (Cluskey and Ramsden, 2001; Piven et al., 1997), or the characterization of axon diameters in the brain white matter (Assaf et al., 2008; Özarslan et al., 2011). The reconstruction techniques are based on the acquisition of diffusionsensitized MR signals, with the acquisition sequence described in (Stejskal and Tanner, 1965), in which a pair of diffusion encoding magnetic field gradient are applied before and after the $180^{\circ}$ pulse. There exists a Fourier relation between the diffusion attenuation $E(\mathbf{q})$

Email address: Emmanuel.Caruyer@inria.fr (Emmanuel Caruyer)

$U R L:$

http://www-sop.inria.fr/members/Emmanuel. Caruyer

(Emmanuel Caruyer)

Preprint submitted to Medical Image Analysis
18

and the EAP

$$
P(\mathbf{r})=\int_{\mathbb{R}^{3}} E(\mathbf{q}) e^{-2 \imath \pi \mathbf{q} \cdot \mathbf{r}} \mathrm{d}^{3} \mathbf{q},
$$

where the wave vector $\mathbf{q}$ is directly related to the applied magnetic field gradient pulse magnitude, direction, and duration.

The diffusion tensor (Basser et al., 1994) is the first model historically proposed to describe the EAP. Despite its wide acceptance into the research and clinical communities, this model restricts the diffusion EAP within the family of Gaussian probability density functions, and is limited for the description of complex tissue structure. Since then, several models and methods were described to extend the results of diffusion tensor, such as high angular resolution diffusion imaging (Tuch, 2004; Descoteaux et al., 2007; Aganj et al., 2010), or higher order tensors (Özarslan and Mareci, 2003). Beyond these approaches, it is possible to reconstruct the model-free diffusion propagator, through Diffusion Spectrum Imaging (DSI) (Wedeen et al., 2005), Diffusion Propagator Imaging (DPI) (Descoteaux et al., 2011), Diffusion Order Transform (Özarslan et al., 2011) or reconstruction in Spherical Polar Fourier (SPF) 
basis (Assemlal et al., 2009). DSI relies on the sampling ${ }_{87}$ of the diffusion signal on a regular Cartesian grid, and 88 reconstructs the EAP through fast Fourier transform. 89 The main limitation of DSI is its huge demand in ac- 90 quisition time, and gradient pulse strength to fulfill the 91 Nyquist conditions (Callaghan, 1991; Tuch, 2004). DPI 92 (Descoteaux et al., 2011) is a more natural method to 93 describe the diffusion signal by a basis of functions so- 94 lution to the 3D Laplace equation by parts. Though this 95 method enables analytical reconstruction of the diffu- 96 sion propagator, it cannot represent the diffusion signal 97 in the whole Q-space. Indeed, DPI represents the sig- 98 nal using the 3D Laplace equation by part (Descoteaux 99 et al., 2011)

$$
E(q \cdot \mathbf{u})=\sum_{l, m}\left[\frac{c_{l, m}}{q^{l+1}}+d_{l, m} q^{l}\right] Y_{l, m}(\mathbf{u}),
$$

where $Y_{l, m}$ is the real, spherical harmonic function. The ${ }_{105}$ basis functions in DPI diverge both for $q \rightarrow 0$ and $q \rightarrow$ $\infty$.

The SPF basis functions instead have a radial profile with a Gaussian-like decay, which is similar to the commonly observed diffusion signal. Besides, it is pos- ${ }_{108}$ sible to recover the EAP (Cheng et al., 2010b) and the ${ }_{109}$ Orientation Distribution Function (ODF) (Cheng et al., 110 2010a) from the coefficients of the signal reconstructed 111 in the SPF basis. The SPF basis is thus a unique, modelfree approach for the reconstruction of the full signal $E$, the estimation of EAP and its derived characteristics. It has been introduced in (Assemlal et al., 2009) together with a regularization method to overcome ill-condition of the estimation problem.

However, the definition of the 3D functions of the SPF basis makes use of the parameterization $\mathbf{q} \in \mathbb{R}^{3}=$ $q \cdot \mathbf{u}$, where $q \in \mathbb{R}^{+}$and $\mathbf{u} \in \mathcal{S}^{2}$. Near the origin, the corresponding $\mathbf{u}$ is not unique, and we show in Section 2.1 that continuity problems near the origin may arise if this parameterization is not used with care. Adding to that, the regularization method introduced in (Assemlal et al., 115 2009) is based on a pair of empirical angular and radial 116 low-pass filters. This regularization method fully relies 117 on the choice of the basis of functions. Besides, its im- 118 plementation requires to tune two separate regulariza- 119 tion weights, which is impractical.

In this work, we propose original and efficient so- ${ }_{121}$ lutions to solve all these important problems. First, 122 we show that continuous functions reconstructed in the classical SPF basis lie in an affine subspace which has a significantly reduced dimension. This means that the signal diffusion could be represented in this subspace ${ }_{123}$ with less coefficients, leading to an estimation process 124 with less measurements than those required when representing the signal in the classical SPF basis. Second, we propose a modified SPF (mSPF) basis, an orthonormal basis for this affine subspace, compatible with the SPF basis, but with reduced dimension and intrinsic continuity near the origin. Thus, the signal reconstructed in the mSPF will satisfy the important continuity constraint. Third, a Laplace regularization functional in the mSPF basis is proposed and minimized for a robust reconstruction of the diffusion signal. The method is analytical and ensures a fast implementation and reconstruction with continuity constraints. The Generalized Cross Validation method is applied to find the unique optimal regularization weight between the regularity of the solution and the data fit. Finally, synthetic and real data are used to illustrate and validate the proposed method. In particular, better reconstruction results with exact continuity constraints are obtained and illustrated in crossing fibers regions.

\section{Theory}

The Spherical Polar Fourier basis was recently introduced in (Assemlal et al., 2009) to reconstruct the diffusion signal in the complete 3D space. The functions $B_{n, l, m}$ of this basis are defined as the product of a radial and an angular function

$$
B_{n, l, m}(q \cdot \mathbf{u})=R_{n}(q) Y_{l, m}(\mathbf{u}) .
$$

$Y_{l, m}$ is the real, symmetric spherical harmonic introduced in (Descoteaux et al., 2006), and the radial function $R_{n}$ is reported below for the record

$$
\begin{aligned}
R_{n}(q) & =\kappa_{n} L_{n}^{1 / 2}\left(\frac{q^{2}}{\zeta}\right) \exp \left(-\frac{q^{2}}{2 \zeta}\right) \\
\kappa_{n} & =\sqrt{\frac{2}{\zeta^{3 / 2}} \frac{n !}{\Gamma(n+3 / 2)}},
\end{aligned}
$$

where $L_{n}^{1 / 2}$ is the generalized Laguerre polynomial, and $\Gamma$ is the Gamma function $\Gamma(z)=\int_{0}^{\infty} t^{z-1} e^{-t} \mathrm{~d} t$. We use $\Omega_{N, L}$ to denote the linear space of functions spanned by the truncated basis $\left\{B_{n, l, m}, n \leq N, l \leq L,|m| \leq l\right\}$. The choice of the scale factor $\zeta$ can be related to the mean diffusivity of the measured data. Several strategies were proposed in Assemlal et al. (2009), here and throughout the experiments, we retain

$$
\zeta=\frac{1}{8 \pi^{2} \tau D},
$$

where $\tau$ is the diffusion time, and $D$ is the mean diffusivity. 


$$
\langle f, g\rangle=\int_{\mathbb{R}^{3}} f(\mathbf{q}) g(\mathbf{q}) \mathrm{d}^{3} \mathbf{q} .
$$

The construction of this basis was motivated by the need ${ }^{174}$ for a complete orthonormal basis of antipodally sym- 175 metric and real functions. Besides, the radial profiles $R_{n} \quad 176$ have a quasi-Gaussian decay, so that even a low radial ${ }_{177}$ truncation order leads to an accurate reconstruction and 178 extrapolation beyond the sampling domain of the dif- 179 fusion weighted attenuation $E(\mathbf{q})$. reviewFrom the reconstruction of the signal in this basis, we can estimate the EAP following Cheng et al. (2010b) and the ODF following Cheng et al. (2010a).

However, a closer look at the functions $B_{n, l, m}$ near ${ }^{18}$ the origin reveals rapid oscillations and a discontinuity. ${ }^{182}$ Moreover, by definition the value of the attenuation $E$ is ${ }^{183}$ equal to 1 when $\mathbf{q}=\mathbf{0}$, but there is nothing in the SPF ${ }^{184}$ basis to impose this. In this work, we show that the sub- ${ }^{185}$ set of functions verifying these properties of continuity ${ }^{186}$ and imposed value at the origin is an affine subspace ${ }^{187}$ of $\Omega_{N, L}$. We propose mSPF, an orthonormal basis for ${ }^{188}$ this subspace, and we give for convenience the relation 189 between this modified SPF (mSPF) basis and the SPF 190 basis $B_{n, l, m}$ introduced in Assemlal et al. (2009).

We also derive the Laplacian regularization functional expression in the mSPF basis, for a robust reconstruction of the diffusion signal. Indeed, the dimension of the basis grows rapidly with the angular and radial orders, and diffusion weighted images have a very low 192 SNR. For the reconstruction of a smooth function, the 193 Laplacian operator is a commonly proposed approach 194 for regularization (Descoteaux et al., 2007). We derive 195 the calculation of the Laplacian operator in the mSPF 196 basis. The method is analytical, which ensures a fast 197 implementation and reconstruction.

In this section, we use indifferently a notation with 199 three indices for the bases elements, such as $B_{n, l, m}$, or 200 a notation with a simple index $i$, convenient for matrix notation. The link between both indexing systems is given by the functions $n(i), l(i)$ and $m(i)$.

\subsection{Continuity in $\Omega_{N, L}$}

Theorem 1. A function $f=\sum_{n, l, m} a_{n, l, m} B_{n, l, m}$ of the $S P F$ basis is continuous if and only if

$$
\forall l>0, \forall|m| \leq l, \sum_{n} a_{n, l, m} R_{n}(0)=0 .
$$

The proof of this theorem is detailed in Appendix A. The linear constraint in Eq. 8 imposes that the polynomial part of $f_{l, m}=\sum_{n} a_{n, l, m} R_{n}$ has no constant term.
This linear constraint can be imposed while estimating the coefficients by constrained least squares estimation. Alternatively, we will derive a new basis of functions to span the subspace of continuous functions. This approach greatly simplifies the Laplace regularization formulation and implementation, as we show in the next section.

In addition to this continuity constraint, we emphasize that the diffusion attenuation signal is defined as $E(\mathbf{q})=S(\mathbf{q}) / S(\mathbf{0})$, and therefore should verify

$$
f(\mathbf{0})=1 .
$$

The set of continuous functions in $\Omega_{N, L}$ verifying Eq. 9 is the solution of an inhomogeneous linear equation, and therefore is an affine subspace of $\Omega_{N, L}$. This affine space is fully characterized by an underlying linear subspace, and an origin. It is underlain by $\Omega_{N, L}^{0}$, the kernel of the associated homogeneous equation $f(\mathbf{0})=0$. As for the origin of the affine subspace, we can choose any solution of Eq. 9. For the sake of simplicity, we choose a simple Gaussian as the origin.

To sum up, any function $f \in \Omega_{N, L}$ verifying the continuity property, together with the property $f(\mathbf{0})=1$ can be expressed as

$$
f(\mathbf{q})=\exp \left(-\frac{\|\mathbf{q}\|^{2}}{2 \zeta}\right)+\sum_{n, l, m} x_{n, l, m} C_{n, l, m}(\mathbf{q}),
$$

where $\left\{C_{n, l, m}\right\}$ is a basis of $\Omega_{N, L}^{0}$, the subspace of continuous functions $f$ in $\Omega_{N, L}$ verifying $f(\mathbf{0})=0$. In the remaining of this section, we give a construction for the orthogonal basis $\left\{C_{n, l, m}\right\}$.

We first construct a basis of radial functions $\left\{F_{n}\right\}$, expressed as $F_{n}(q)=\chi_{n} q^{2} / \zeta P_{n}\left(q^{2} / \zeta\right) \exp \left(-q^{2} / 2 \zeta\right)$. This verifies $F_{n}(0)=0$; the polynomials $P_{n}$ and the normalization constant $\chi_{n}$ are to determine, provided that the following orthogonality property is fulfilled

$$
\left\langle F_{n}, F_{p}\right\rangle_{\mathbb{R}^{3}}=\int_{0}^{\infty} F_{n}(q) F_{p}(q) q^{2} \mathrm{~d} q=\delta_{n, p} .
$$

The substitution $u=q^{2} / \zeta$ in Eq. 11 gives

$$
\int_{0}^{\infty} \chi_{n} \chi_{m} \frac{\zeta^{3 / 2}}{2} P_{n}(u) P_{p}(u) u^{5 / 2} e^{-u} \mathrm{~d} u=\delta_{n, p} .
$$

$$
F_{n}(q)=\chi_{n} \frac{q^{2}}{\zeta} L_{n}^{5 / 2}\left(\frac{q^{2}}{\zeta}\right) e^{-q^{2} / 2 \zeta},
$$


and the normalization constant

$$
\chi_{n}=\sqrt{\frac{2}{\zeta^{3 / 2}} \frac{n !}{\Gamma(n+7 / 2)}} .
$$

The diffusion attenuation $E(\mathbf{q})-\exp \left(-\|\mathbf{q}\|^{2} / 2 \zeta\right)$ is reconstructed through the functions

$$
C_{n, l, m}(\mathbf{q})=F_{n}(\|\mathbf{q}\|) Y_{l, m}\left(\frac{\mathbf{q}}{\|\mathbf{q}\|}\right) .
$$

The family of functions $\left\{C_{n, l, m}, n=0 \ldots N-1, l={ }^{247}\right.$ $0 \ldots L, m=-l \ldots l\}$ is the modified SPF (mSPF) basis, ${ }^{248}$ an orthonormal basis of $\Omega_{N, L}^{0}$.

The coefficients $x_{n, l, m}$ are estimated by minimization of the squared error criterion $\|\mathbf{y}-\mathbf{H x}\|^{2}$, where $\mathbf{y}$ is the vector of observations $y_{k}=E\left(\mathbf{q}_{k}\right)-\exp \left(-\left\|\mathbf{q}_{k}\right\|^{2} / 2 \zeta\right)$ measured at wave vectors $\mathbf{q}_{k}$. The observation matrix has entries $H_{k, i}=C_{n(i), l(i), m(i)}\left(\mathbf{q}_{k}\right)$.

This new space has a substantially reduced dimension: $\operatorname{dim}\left(\Omega_{N, L}\right)=(N+1) \cdot L(L+1) / 2$, whereas $\operatorname{dim}\left(\Omega_{N, L}^{0}\right)=N \cdot L(L+1) / 2$. This dimension reduc- ${ }_{254}$ tion comes from the two systems of linear constraints ${ }_{25}$ of Eq. $8\left(L(L+1) / 2-1\right.$ equations), and Eq. 9 (1 equa- ${ }_{256}$ tion). As an example, when the angular truncation order ${ }_{257}$ $L=4$ is used, the reconstruction in $\Omega_{N, L}^{0}$ requires 15 less ${ }_{258}$ coefficients, to represent the same signal. This simpli- ${ }_{259}$ fies the implementation, reduces the demand in storage ${ }_{260}$ capacity, and improves computational efficiency.

\subsection{Link with the SPF basis}

In this section we give the link between SPF and 265 mSPF bases. This relationship is useful as SPF (Assemlal et al., 2009) is a now a state-of-the-art method in diffusion MRI. We can therefore reconstruct the ensem- 266 ble average propagator (EAP) following Cheng et al. ${ }^{267}$ (2010b), the orientation distribution function (ODF) following Cheng et al. (2010a), or the apparent fiber popu- 268 lation dispersion following Assemlal et al. (2011). The 269 SPF basis is built on Laguerre polynomials $L_{n}^{1 / 2}$ while ${ }_{270}$ we use $L_{n}^{5 / 2}$ in this work. Using the recurrence relations ${ }_{271}$ between Laguerre polynomials detailed in (Abramowitz and Stegun, 1970, p. 783), we have:

$$
F_{n}(q)=\sum_{i=0}^{n} \frac{3 \chi_{n}}{2 \kappa_{i}} R_{i}(q)-\frac{(n+1) \chi_{n}}{\kappa_{n+1}} R_{n+1}(q) .
$$

If the function $f(\mathbf{q})=E(\mathbf{q})-\exp \left(-\|\mathbf{q}\|^{2} / 2 \zeta\right)$ is expressed in this basis, $f(\mathbf{q})=\sum x_{n, l, m} C_{n, l, m}(\mathbf{q})$, then the coefficients $a_{n, l, m}$ of $E$ in the SPF basis are obtained by
$\mathbf{a}=\mathbf{M x}+\mathbf{a}^{0}$, where

$$
M_{i j}=\delta_{l(i), l(j)} \delta_{m(i), m(j)} \cdot \begin{cases}\frac{3 \chi_{n(j)}}{2 \kappa_{n(i)}} & n(i) \leq n(j) \\ -\frac{n(i) \chi_{n(j)}}{\kappa_{n(i)}} & n(i)=n(j)+1 \\ 0 & n(i)>n(j)+1\end{cases}
$$

and $\mathbf{a}^{0}=\left[\begin{array}{lllll}\sqrt{4 \pi} / \kappa_{0} & 0 & 0 & \ldots\end{array}\right]^{\mathrm{T}}$, as $\exp \left(-\|\mathbf{q}\|^{2} / 2 \zeta\right)=$ $\sqrt{4 \pi} / \kappa_{0} B_{0,0,0}(\mathbf{q})$.

$\mathbf{M}$ is the change-of-basis matrix from mSPF to SPF, two orthonormal bases. Therefore, this matrix is orthogonal: the orthogonal projection of any function in $\Omega_{N, L}$, represented by its coefficients $\mathbf{a}$ in the SPF basis, onto the subspace $\Omega_{N, L}^{0}$ has coefficients $\mathbf{x}=\mathbf{M}^{\mathrm{T}} \mathbf{a}$.

\subsection{Laplace regularization in the $M S P F$ basis}

In this section, we propose to introduce a regularization term in the fitting procedure. We choose as a regularization functional

$$
U(\mathbf{x})=\int_{\mathbb{R}^{3}}\left|\Delta E_{\mathbf{x}}(\mathbf{q})\right|^{2} \mathrm{~d}^{3} \mathbf{q},
$$

where $E_{\mathbf{x}}(\mathbf{q})=\exp \left(-\left\|\mathbf{q}_{k}\right\|^{2} / 2 \zeta\right)+\sum_{i} x_{i} C_{i}(\mathbf{q})$ is the reconstructed signal. This continuous operator is rotational invariant, and independent on the choice of a specific basis. Besides, the Laplace operator was already applied successfully for several applications ranging from natural image denoising (You and Kaveh, 2000; Chan and Shen, 2005) to diffusion MRI analysis (Descoteaux et al., 2007; Koay et al., 2009; Descoteaux et al., 2010).

We minimize $\|\mathbf{y}-\mathbf{H x}\|^{2}+\lambda U(\mathbf{x})$, where the observations are $y_{k}=E\left(\mathbf{q}_{k}\right)-\exp \left(-\left\|\mathbf{q}_{k}\right\|^{2} / 2 \zeta\right)$ and $\mathbf{H}$ is the observation matrix. In this section, we write the Laplace penalization as a quadratic form

$$
U(\mathbf{x})=\left(\mathbf{x}-\mathbf{x}_{0}\right)^{\mathrm{T}} \boldsymbol{\Lambda}\left(\mathbf{x}-\mathbf{x}_{0}\right)+U_{0} .
$$

Hence the penalized least squares has a unique minimum

$$
\hat{\mathbf{x}}=\mathbf{x}_{0}+\left(\mathbf{H}^{\mathrm{T}} \mathbf{H}+\lambda \mathbf{\Lambda}\right)^{-1}\left(\mathbf{y}-\mathbf{H} \mathbf{x}_{0}\right) .
$$

In what follows, we give explicit directions how to compute the matrix $\boldsymbol{\Lambda}$ and the vector $\mathbf{x}_{0}$.

When $E_{\mathbf{x}}(\mathbf{q})-\exp \left(-\left\|\mathbf{q}_{k}\right\|^{2} / 2 \zeta\right)$ is expressed in the mSPF basis with coefficients $x_{i}$,

$$
\begin{aligned}
U(\mathbf{x})= & \int_{\mathbb{R}^{3}}\left(\sum_{i} x_{i} \Delta C_{i}(\mathbf{q})+\Delta e^{-\left\|\mathbf{q}_{k}\right\|^{2} / 2 \zeta}\right)^{2} \mathrm{~d}^{3} \mathbf{q} \\
= & \sum_{i} \sum_{j} x_{i} x_{j} \int_{\mathbb{R}^{3}} \Delta C_{i}(\mathbf{q}) \cdot \Delta C_{j}(\mathbf{q}) \mathrm{d}^{3} \mathbf{q} \\
& +2 \sum_{i} x_{i} \int_{\mathbb{R}^{3}} \Delta C_{i}(\mathbf{q}) \cdot \Delta e^{-\left\|\mathbf{q}_{k}\right\|^{2} / 2 \zeta} \mathrm{d}^{3} \mathbf{q} \\
& +\ldots
\end{aligned}
$$


The constant term is discarded since it plays no role in 295 the minimization. Thus we have the quadratic form of 296 Eq. 18, where

$$
\Lambda_{i j}=\int_{\mathbb{R}^{3}} \Delta C_{i}(\mathbf{q}) \cdot \Delta C_{j}(\mathbf{q}) \mathrm{d}^{3} \mathbf{q}
$$

$$
v_{i}=\int_{\mathbb{R}^{3}} \Delta C_{i}(\mathbf{q}) \cdot \Delta e^{-\left\|\mathbf{q}_{\mathbf{k}}\right\|^{2} / 2 \zeta} \mathrm{d}^{3} \mathbf{q}
$$

The Laplace operator $\Delta$ can be written in spherical coordinates, with the Laplace-Beltrami operator $\Delta_{\mathrm{b}}, \quad{ }_{307}$

$$
\begin{aligned}
\Delta C_{n, l, m}(q \mathbf{u})= & \chi_{n}\left(\frac{1}{q^{2}} \frac{\partial}{\partial q}\left(q^{2} F_{n}^{\prime}(q)\right) Y_{l, m}(\mathbf{u})\right. \\
& \left.+\frac{F_{n}(q)}{q^{2}} \Delta_{\mathrm{b}} Y_{l, m}(\mathbf{u})\right)
\end{aligned}
$$

Since the spherical harmonics are eigenfunctions of the Laplace-Beltrami operator with eigenvalue $-l(l+1)$, we have

$\Delta C_{n, l, m}(q \mathbf{u})=\chi_{n}\left(F_{n}^{\prime \prime}(q)+2 \frac{F_{n}^{\prime}(q)}{q}-\frac{l(l+1) F_{n}(q)}{q^{2}}\right) Y_{l, m}(\mathbf{u}){ }_{315}^{314}$

As the spherical harmonics form an orthonormal basis ${ }_{317}$ for the canonical dot product on $\mathcal{S}^{2}$, the entries of the ${ }_{318}$ matrix $\boldsymbol{\Lambda}$ are

$$
\Lambda_{i, j}=\delta_{l(i), l(j)} \delta_{m(i), m(j)} \int_{0}^{\infty} h_{i}(q) h_{j}(q) \mathrm{d} q,
$$

where

$$
h_{i}=\chi_{n(i)}\left(q F_{n(i)}^{\prime \prime}+2 F_{n(i)}^{\prime}-\frac{l(i)(l(i)+1)}{q} F_{n(i)}\right) .
$$

Similarly, the vector $\mathbf{v}$ has entries

$$
v_{i}=\delta_{l(i), 0} \delta_{m(i), 0} \int_{0}^{\infty} h_{i}(q) \cdot\left(\frac{q^{3}}{\zeta^{2}}-\frac{3 q}{\zeta}\right) \exp \left(-\frac{q^{2}}{2 \zeta}\right) \mathrm{d} q .
$$

The computation of the integrals in Eq. B.1 and 28 is analytical and needs no numerical integration. It is described in details in Appendix B.

\section{Material and methods}

\subsection{Optimal regularization parameters}

We adopted the Generalized Cross Validation (GCV) algorithm (Craven and Wahba, 1985) to find the regu- 336 larization weight $\lambda$ which guarantees the best balance ${ }_{337}$ between the smoothness of the reconstruction, and the ${ }_{338}$ data fit. This algorithm, as well as the L-curve method (Hansen, 2000), have already been applied successfully for other applications in Q-ball diffusion MRI (Koay et al., 2009; Descoteaux et al., 2010, 2007). The GCV method has the major advantage to be generalizable to the situation where there is more than one $\lambda$ parameter to optimize. It is the case in (Assemlal et al., 2009), where there are two regularization matrices $\mathbf{N}$ and $\mathbf{L}$, which act respectively as radial and angular low-pass filters, with corresponding weights $\lambda_{N}$ and $\lambda_{L}$.

The GCV method is based on a one-fold cross validation: among $K$ samples, we use $K-1$ samples to fit the model parameters, and predict the $K$-th left-apart sample. The process is repeated $K$ times, and the mean prediction error is the value we want to minimize. Fortunately, the mean prediction error, called the GCV function, has a simple expression

$$
\operatorname{GCV}(\lambda ; \mathbf{y})=\frac{\left\|\mathbf{y}-\hat{\mathbf{y}}_{\lambda}\right\|^{2}}{K-\operatorname{Tr}\left(\mathbf{S}_{\lambda}\right)},
$$

which makes this method very efficient. The matrix $\mathbf{S}_{\lambda}=\mathbf{H}\left(\mathbf{H}^{\mathrm{T}} \mathbf{H}+\lambda \mathbf{\Lambda}\right)^{-1} \mathbf{H}^{\mathrm{T}}$ is the smoother matrix, and $\hat{\mathbf{y}}_{\lambda}=\mathbf{S}_{\lambda} \mathbf{y}$. With the GCV method, it is possible to adapt the regularization parameters to the data. However, there is no analytical solution for the minimization of the GCV function and for computational efficiency, we compute the optimal $\lambda$ parameters once. This choice is validated in the next section, and results show it is indeed a good compromise.

\subsection{Synthetic and real data}

We simulate diffusion weighted measurements with a multi-compartment Gaussian model

$$
E(\mathbf{q})=\sum_{p=1}^{P} \omega_{p} \exp \left(-2 \pi \tau \mathbf{q}^{\mathrm{T}} \mathbf{D}_{p} \mathbf{q}\right)
$$
the relative compartment size and $\mathbf{D}_{p}$ the corresponding diffusion tensor. The diffusion weighted signal is corrupted by Rician noise, with controlled variance parameter $\sigma$. Using this diffusion model locally, we created a synthetic diffusion field simulating a sin-shaped and a straight fiber, crossing each other at $90^{\circ}$.

The wave vectors $\mathbf{q}_{k}$ for synthesis are arranged on 3 shells, with the strategy recently proposed in (Caruyer et al., 2011a,b). In short, this method is a generalization of the electrostatic repulsion, introduced in (Jones et al., 1999) for single Q-shell experiment design, to the

\footnotetext{
The experiments on real data were carried out on the publicly available phantom (Poupon et al., 2008; Fillard
} 
et al., 2011) which served as the data for a tractography contest, held at the DMFC MICCAI workshop, London (2009). The diffusion signal was sampled on 3 Q-shells, with $b$-values ranging from 650 to $2000 \mathrm{~s} \cdot \mathrm{mm}^{-2}$, and 64 directions per shell.

For the experiments, we compare the diffusion signal, the ensemble average propagator (EAP) reconstructed from the SPF coefficients by the method in (Cheng et al., 2010b), and the orientation distribution function (ODF) reconstructed in constant solid angle, implementing the technique in (Cheng et al., 2010a).

\subsection{Exact and empirical continuity constraints}

We presented in Section 2.1 a linear constraint to impose the continuity of the reconstructed signal. An alternate solution proposed in (Cheng et al., 2010b) is to artificially add $P$ virtual data points $\mathbf{q}_{k}, k=K+1 \ldots P$ close to zero, verifying $E\left(\mathbf{q}_{k}\right)=1$. As $P$ goes to infinity, it is possible to show that the solution of this system tends to the exact solution (see Golub and Van Loan, 1983, pp. 410-412). We study the convergence of this empirical continuity approach. As a measure of discontinuity of the reconstructed signal $\hat{E}$ about $\mathbf{0}$, we define $d(\hat{E})$ the difference between extremal values of the set $\left\{\lim _{q \rightarrow 0^{+}} \hat{E}(q \mathbf{u}), \mathbf{u} \in \mathcal{S}^{2}\right\}$. We also compare the relative difference between the solution $\mathbf{c}_{\mathrm{AC}}$ of the least squares problem with analytical constraint, and the so- 391 lution $\mathbf{c}_{\mathrm{EC}}(P)$ of the system with empirical constraint ${ }_{392}$ with $P$ virtual measurements.

\section{Results and discussion}

\subsection{Continuity constraint}

We compare the solution $\mathbf{c}_{\mathrm{AC}}$ and $\mathbf{c}_{\mathrm{EC}}(P)$, for a sin- 399 gle Gaussian distribution. To focus on the continuity 400 constraint, we do not impose any other kind of regular- ${ }_{401}$ ization. The signal is corrupted by Rician noise, with ${ }_{402}$ corresponding SNR $=25$. An example of signal and its 403 reconstruction is reported on Fig. 1.

We evaluate the difference of the signal reconstructed 405 with exact continuity constraint and with empirical con- 406 straint. We plot on Fig. 2 the relative squared difference ${ }_{407}$ between the coefficients estimated with a strict continu- 408 ity constraint, $\hat{\mathbf{c}}_{\mathrm{AC}}$, and the coefficients estimated with 409 an empirical continuity constraint, $\hat{\mathbf{c}}_{\mathrm{EC}}$. The conver- 410 gence is pretty fast, and $P=60$ virtual measurements 411 give good results. This confirms the intuition in (Cheng ${ }_{412}$ et al., 2010b); however the minimum number of virtual ${ }_{413}$ measurements $P$ for an acceptable accuracy heavily de- 414 pends on the angular order of the SPF basis, as reported 415

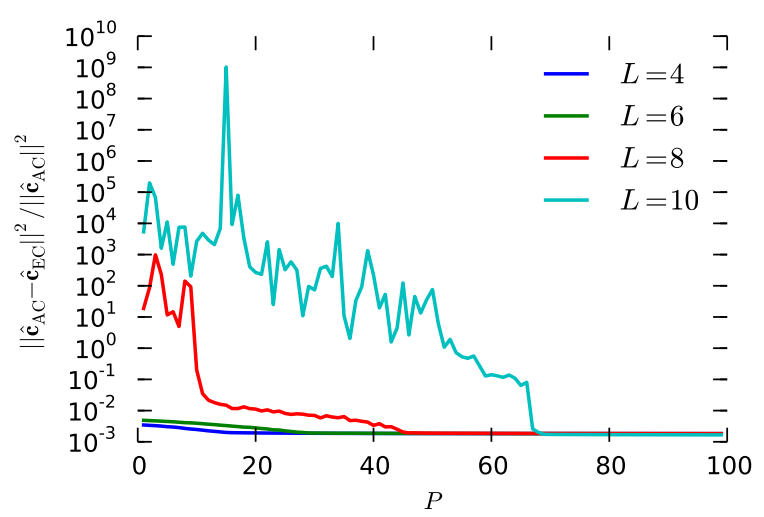

Figure 2: Relative difference between reconstruction with a strict continuity constraint, and reconstruction with a loose continuity constraint. Results on a synthetic Gaussian diffusion signal, from $K=$ 150 measurements on 3 Q-shells, plus $P$ virtual measurements at $\mathbf{q}=\mathbf{0}$, for various angular orders $L$ of the SPF basis. Depending on the radial order, the number of additional measurements needed for an accurate reconstruction may become huge, and really impractical.

on Fig. 2. This makes this empirical solution impractical. Besides, discontinuity is not strictly imposed: as experimented and reported on Fig. 3, the value of $d(\hat{E})$ remains unacceptably high while we impose the value on $P=150$ virtual measurements.

\subsection{Laplace regularization}

Laplace regularization was implemented in the $\mathrm{MSPF}$ basis, and we compare it with separate LaplaceBeltrami and radial low-pass filter, proposed in (Assemlal et al., 2009). The GCV function is significantly lower for the optimal Laplace regularization (Table 1). This result suggests that Laplace regularization is more suitable than separate Laplace-Beltrami and radial low-pass filtering. Furthermore, the optimal $\lambda_{\Lambda}$ parameter does not vary much from one diffusion model to another. We can therefore select a unique $\lambda_{\Lambda}$ parameter for the regularization of a whole volume.

The regularization also impacts on the extrapolation capacity of the method. Hardware limitations often restrict the sampling to a bounded region in the Q-space. Increasing the radial order of the mSPF basis will allow better signal reconstruction within the sampled area of the Q-space. It might however introduce undesirable oscillations outside this area, as reported on Fig. 4, where the radial truncation order was set to $N=5$. Adding a regularization constraint greatly improves the extrapolation of the diffusion signal. Laplace regularization performs slightly better in this task, though a more complete study, involving real data and outside the scope of this paper, should be carried out to further validate this. 


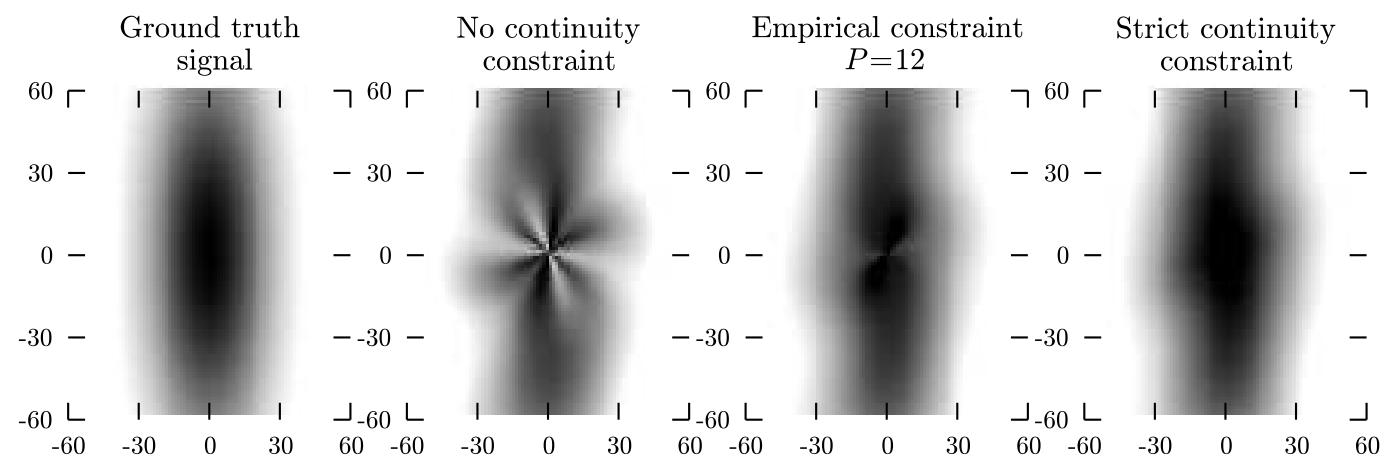

Figure 1: Diffusion signal corresponding to a single fiber oriented along the $x$-axis, reconstructed from 120 samples in the Q-space. The signal is shown on the $\left(q_{x}, q_{y}\right)$-plane, and the grey levels correspond to signal range from 0.0 (white) to 1.0 (black). $q$ values are understood in $\mathrm{mm}^{-1}$. This illustrates the discontinuity at the origin inherent to the SPF basis, and how the reconstruction in mSPF solves this problem.

\begin{tabular}{l|ccc|} 
& 1 fiber & 2 fibers, $90^{\circ}$ & 2 fibers, $60^{\circ}$ \\
\hline$\left(\lambda_{L}^{0}, \lambda_{N}^{0}\right)$ & $\left(4.0 \cdot 10^{-7}, 8.1 \cdot 10^{-9}\right)$ & $\left(3.2 \cdot 10^{-1}, 1.2 \cdot 10^{-8}\right)$ & $\left(5.1 \cdot 10^{-8}, 5.5 \cdot 10^{-8}\right)$ \\
$\mathrm{GCV}_{L, N}^{0}$ & $5.7 \cdot 10^{-1}$ & $3.4 \cdot 10^{-1}$ & $4.8 \cdot 10^{-1}$ \\
\hline$\lambda_{\Lambda}^{0}$ & $1.6 \cdot 10^{-1}$ & $1.7 \cdot 10^{-1}$ & $2.4 \cdot 10^{-1}$ \\
$\mathrm{GCV}_{\Lambda}^{0}$ & $5.3 \cdot 10^{-1}$ & $3.1 \cdot 10^{-1}$ & $4.2 \cdot 10^{-1}$ \\
\hline
\end{tabular}

Table 1: Optimal $\lambda$ parameters and corresponding GCV minimum, for various synthetic diffusion models. The sampling consists in 200 diffusion weighted measurements on 3 Q-shells, with a max $b$-value of $3000 \mathrm{~s} \cdot \mathrm{mm}^{-2}$. Radial and angular orders were set to 5 and 6 , respectively. 1 st row: separate Laplace-Beltrami and radial low-pass filter smoothing, 2nd row: Laplace regularization.

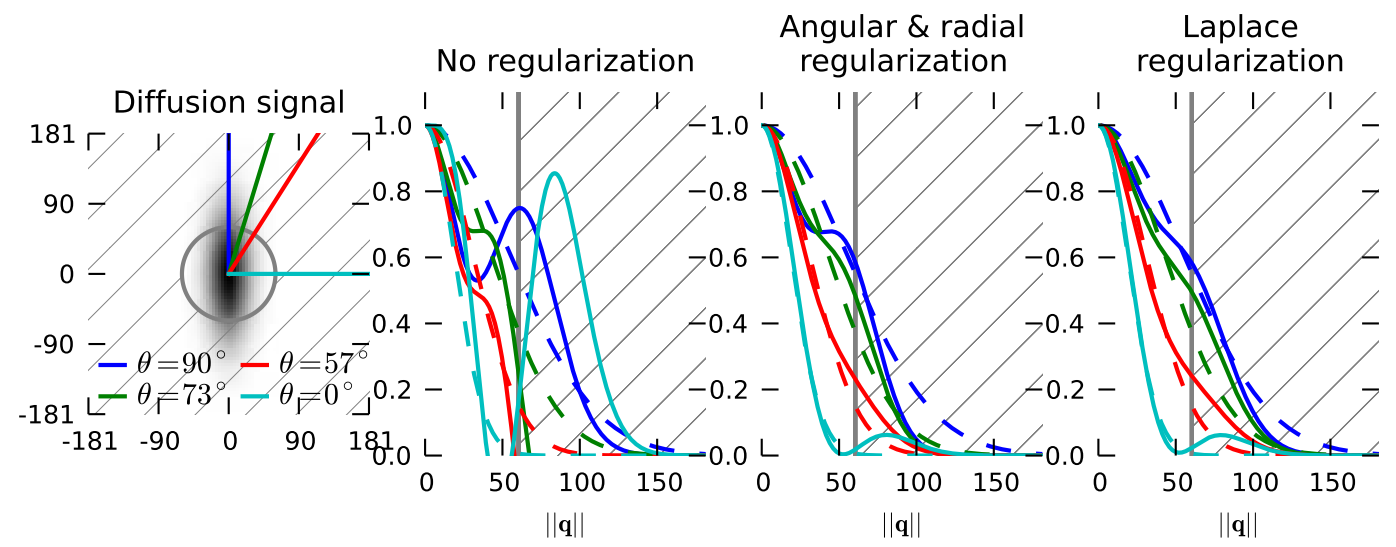

Figure 4: Reconstruction and extrapolation of a diffusion signal, for a Gaussian diffusion model, from 120 measurements on 3 Q-shells. We plot the reconstructed (solid lines) and ground truth (dashed lines) radial profiles of the signal on selected lines in the Q-space. The maximum $q$ value of the sampling scheme was set to $60 \mathrm{~mm}^{-1}$, the hatched area represents the no-sample area. We compare the reconstruction without regularization, with separate Laplace-Beltrami and radial filter, and with Laplace regularization. Laplace regularization performs better in smoothing radial profiles, and we avoid oscillations outside the sampling area. 


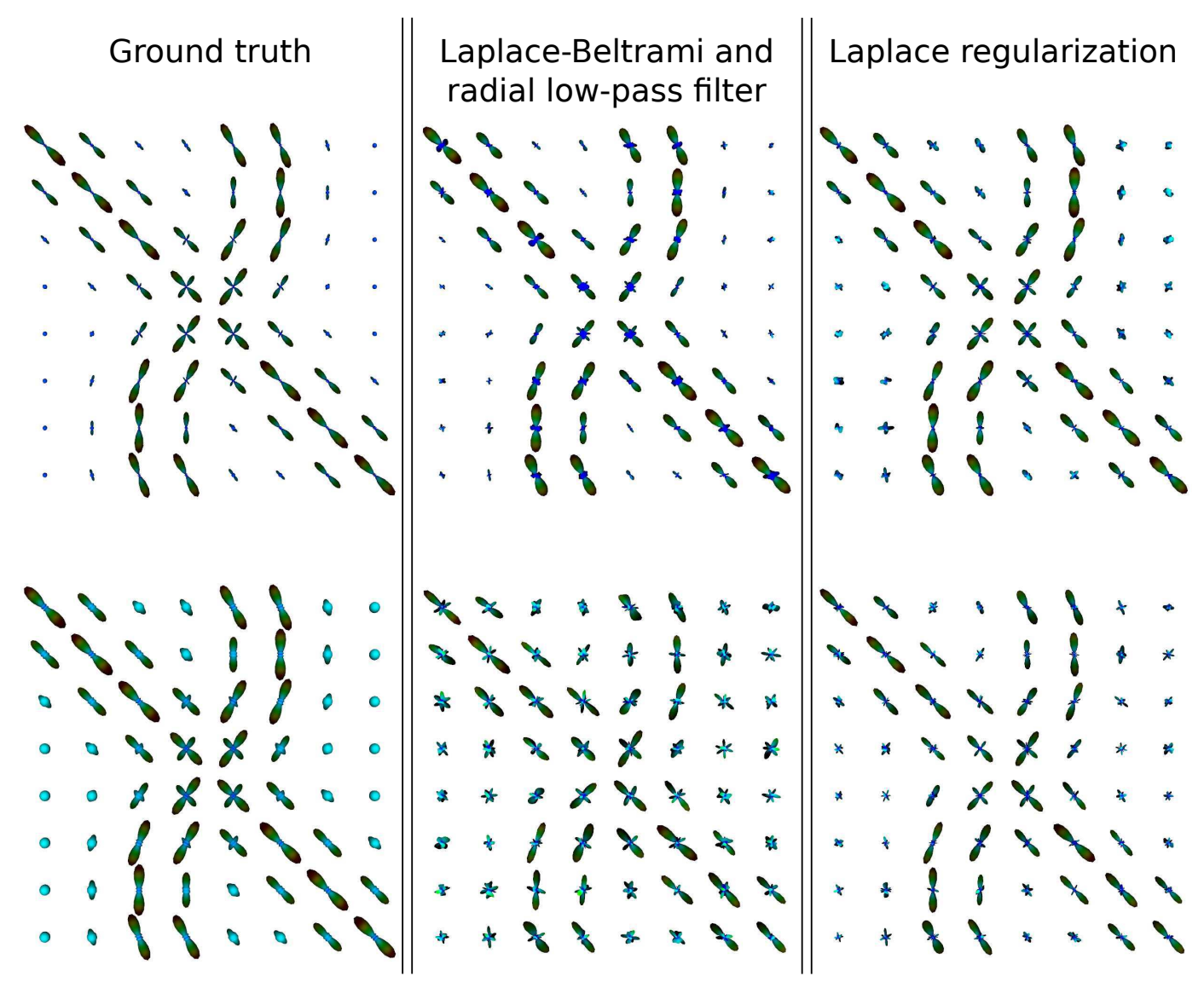

Figure 5: Reconstruction of a diffusion propagator field, from 120 measurements on 3 shells (max $b$-value was $3000 \mathrm{~s} \cdot \mathrm{mm}^{-2}$ ). We compare the diffusion EAP profile (top row) $P\left(r_{0} \mathbf{u}\right)$, for $r_{0}=15 \mu \mathrm{m}$, and the diffusion ODF $\psi(\mathbf{u})$ (bottom row). Fiber crossing are better resolved with Laplace regularization, and isotropic regions are smoother. 


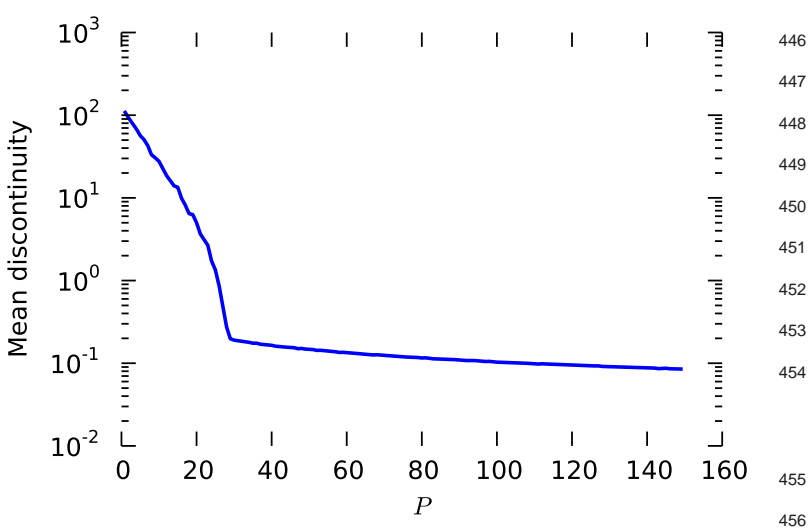

Figure 3: Discontinuity, measured about the origin, of a synthetic Gaussian diffusion signal, reconstructed from $K$ measurements on 3 Q-shells, plus $P$ virtual measurements at $\mathbf{q}=\mathbf{0}$. The discontinuity remains very high, even for a large number of additional, virtual measurements $(P=150)$.

We also compare the reconstruction with both regu- ${ }_{462}$ larization constraints on our synthetic diffusion field in ${ }_{463}$ Fig. 5. Laplace regularization performs better in cross- 464 ing fiber regions, and the results show better directional ${ }_{465}$ coherence. Besides, in isotropic regions, the reconstructed ODFs have a smoother profile than with separate Laplace-Beltrami and radial filtering.

Similar results are obtained on the real data experiment, depicted on Fig. 6. We have overlaid the ground truth fiber orientations, as provided by Fillard et al. (2011). The reconstruction results with optimal Laplace regularization show slighly sharper EAP and ODF profiles. We acknowledge that the reconstruction of this dataset was very challenging, due to the low anisotropy of the signal.

\section{Conclusions}

We have proposed a novel orthonormal basis for the reconstruction of the diffusion signal in the complete 3D Q-space, based on Gaussian-Laguerre functions. This new method enables the reconstruction of a continuous signal, with known value at the origin. This mathematical constraint results in a dimension reduction with re- ${ }^{472}$ spect to the SPF basis, and a better reconstruction of 473 the diffusion signal at the same sampling rate. This 474 also greatly simplifies the reconstruction method, and 475 reduces the associated computational cost as the conti- 476 nuity constraint is naturally imposed. The MSPF basis 477 is presented with its linear relation to the SPF basis for 478 convenience, so that the methods of SPF imaging di- 479 rectly transpose to $\mathrm{mSPF}$.
We also derive a regularization functional based on the Laplace operator, together with its analytical expression in the mSPF basis. This is shown to be mathematically and practically better than separate LaplaceBeltrami and radial low-pass filtering. The experiments on simulations and real data show good results, for the reconstruction and extrapolation of the radial profile. The angular profile reconstruction is more robust to noise, and better detection of fiber crossing is reported.

\section{Appendix A. Necessary and sufficient condition for the continuity}

In this appendix, we give a proof of Theorem 1, relative to the continuity of a function $f \in \Omega_{N, L}$, expressed as a sum of SPF functions.

\section{Appendix A.1. Necessary condition}

A necessary condition for the continuity of the function $f$ is that the restriction of $f$ to any line in $\mathbb{R}^{3}$ must be continuous about 0 . For $\mathbf{u} \in S^{2}$ and $q \in \mathbb{R}$, we note $f_{\mathbf{u}}(q)=f(q \mathbf{u})$ the restriction of $f$ to the line of direction u.

$$
\begin{aligned}
& \lim _{q \rightarrow 0^{+}} f_{\mathbf{u}}(q)=f_{\mathbf{u}}(0)=f(\mathbf{0}) \\
\Rightarrow & \sum_{n, l, m} a_{n, l, m} R_{n}(0) Y_{l, m}(\mathbf{u})=f(\mathbf{0}) \\
\Rightarrow & \sum_{l, m}\left(\sum_{n=0}^{N} a_{n, l, m} R_{n}(0)\right) Y_{l, m}(\mathbf{u})=f(\mathbf{0}) .
\end{aligned}
$$

Eq. A.3 must hold for any $\mathbf{u} \in S^{2}$. The left hand part is written as a sum of spherical harmonic functions, while the right hand part does not depend on $\mathbf{u}$.

The only constant function in the Spherical Harmonics basis is $Y_{0,0}$. Hence all the spherical harmonic coefficients in Eq. A.3 must be zero, except for $l=m=0$.

$$
\forall l>0, \forall m \text { s. t. }|m| \leq l, \sum_{n=0}^{N} a_{n, l, m} R_{n}(0)=0
$$

\section{Appendix A.2. Sufficient condition}

Now we show that if the necessary condition in Eq. A. 4 is met, then the function $f$ is continuous about 0. We can write $f$ as a finite sum of functions $f_{l, m}=$ $\sum_{n} a_{n, l, m} B_{n, l, m}$. If we prove the continuity of $f_{l, m}$, for any $0 \leq l \leq L$ and any $-l \leq m \leq l$, then by linearity we prove the continuity of $f$.

The continuity of $f_{00}$ is direct, as the Gauss-Laguerre functions are continuous and $Y_{00}$ is constant. Next, we 


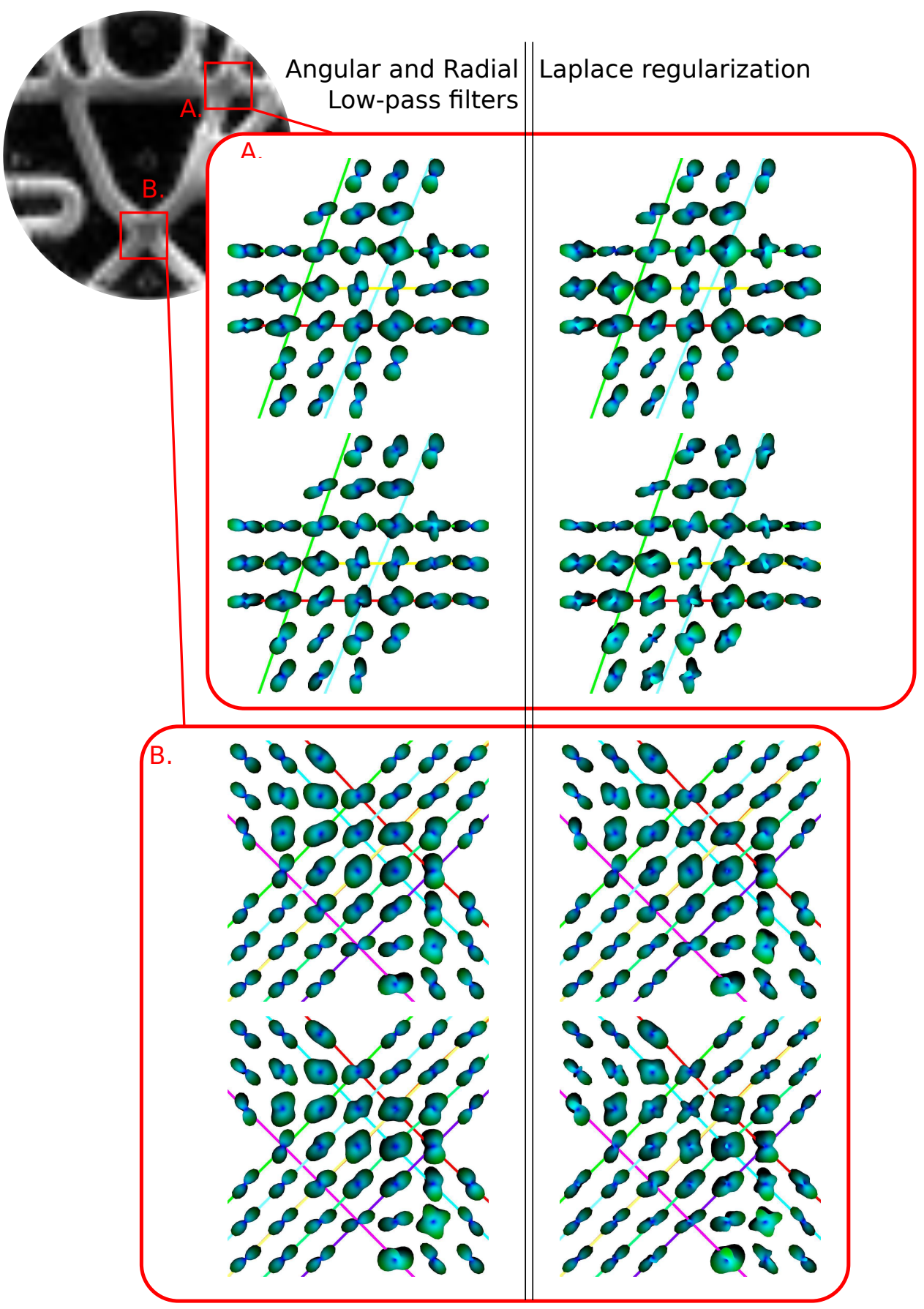

Figure 6: Diffusion ODF and EAP profiles reconstructed from the diffusion MRI data of the fiber cup. Zooms on crossing regions A and B are displayed. Within each block: EAP profile $P\left(r_{0} \mathbf{u}\right)$, for $r_{0}=17 \mu \mathrm{m}$ (top row) and diffusion ODF reconstructed in constant solid angle $\psi(\mathbf{u})$ (bottom row). The left column corresponds to a reconstruction with separate angular and radial low-pass filters, while the right column is the reconstruction with Laplace regularization. The EAP profiles and ODF reconstructed with Laplace regularization are somehow sharper in crossing regions. 
This is true for $\epsilon^{\prime}=\epsilon /\left\|Y_{l, m}\right\|_{\infty}$. Besides,

$$
\forall \mathbf{u} \in S^{2}, \frac{\left|Y_{l, m}(\mathbf{u})\right|}{\left\|Y_{l, m}\right\|_{\infty}} \leq 1,
$$

484 hence

$$
\begin{aligned}
& \forall \mathbf{u} \in S^{2},|q|<\alpha \Rightarrow \\
& \left\|\sum_{n=0}^{N} a_{n, l, m} R_{n}(q)\right\| \frac{\left\|Y_{l, m}(\mathbf{u})\right\|}{\left\|Y_{l, m}\right\|_{\infty}}<\frac{\epsilon}{\left\|Y_{l, m}\right\|_{\infty}} .
\end{aligned}
$$

485

486

491 we can write $\forall \epsilon^{\prime}>0, \exists \alpha>0$ such that

$$
|q|<\alpha \Rightarrow\left\|\sum_{n=0}^{N} a_{n, l, m} R_{n}(q)\right\|<\epsilon^{\prime} .
$$

This proves the continuity of $f_{l, m}$ about $\mathbf{0}$, and by linearity the continuity of $f$.

\section{Appendix B. Laplace regularization matrix}

In this appendix, we derive the general expression of ${ }^{51}$ the Laplace regularization matrix $\boldsymbol{\Lambda}$ in the MSPF basis. The entries of the matrix $\boldsymbol{\Lambda}$ are

$$
\Lambda_{i, j}=\delta_{l(i), l(j)} \delta_{m(i), m(j)} \int_{0}^{\infty} h_{i}(q) h_{j}(q) \mathrm{d} q,
$$

$$
h_{i}=\chi_{n(i)}\left(q F_{n(i)}^{\prime \prime}+2 F_{n(i)}^{\prime}-\frac{l(i)(l(i)+1)}{q} F_{n(i)}\right) .
$$

The function $h_{i}$ can be written as

$$
h_{i}(q)=\chi_{n(i)} \frac{q}{\zeta} \exp \left(-\frac{q^{2}}{2 \zeta}\right) G_{n(i), l(i)}\left(\frac{q^{2}}{\zeta}\right),
$$

where $G_{n, l}=\sum_{k} g_{k}^{n, l} X^{k}$ is a polynomial. It is hard ${ }^{53}$ to express the coefficients $g_{k}^{n, l}$ in a compact form. In- ${ }_{533}^{532}$ stead of manually deriving these coefficients, we com- ${ }^{534}$ pute them using polynomial algebra facilities, provided ${ }^{535}$ in the SciPy library (Jones et al., 2001) in Python ${ }^{\mathrm{TM}}$. ${ }_{537}^{536}$ The coefficients $g_{k}^{n, l}$ are algebraically computed on de- ${ }_{538}^{537}$ mand as it involves simple operation on polynomials: ${ }^{539}$ derivation and addition. The first coefficients are tabu- ${ }^{540}$ lated here for convenience.

\begin{tabular}{|c|c|c|c|}
\hline$k$ & $G_{0, l}$ & $G_{1, l}$ & $G_{2, l}$ \\
\hline 0 & $6-l(l+1)$ & $7(3-l(l+1) / 2)$ & $15.75(3-l(l+1) / 2)$ \\
\hline 1 & -7 & $-44.5+l(l+1)$ & $-145.125+4.5 l(l+1)$ \\
\hline 2 & 1 & 14.5 & $78.375-l(l+1) / 2$ \\
\hline 3 & & -1 & -12 \\
\hline 4 & & & 0.5 \\
\hline
\end{tabular}

Hence the integrand $h_{i}(q) h_{j}(q)$ can be written as

where $T_{i, j}(X)$ is the polynomial $X G_{n(i), l(i)}(X) G_{n(j), l(j)}(X)$. The coefficients $a_{k}^{i, j}$ of $T_{i, j}$ are simply obtained from the coefficients of $G_{n(i), l(i)}$ and $G_{n(j), l(j)}$. Therefore, the entries of the regularization matrix are

$$
\begin{aligned}
\Lambda_{i, j} & =\frac{\chi_{n(i)} \chi_{n(j)}}{\zeta} \sum_{k=0}^{d} a_{k}^{i, j} \int_{0}^{\infty} \exp \left(-q^{2} / \zeta\right)\left(\frac{q^{2}}{\zeta}\right)^{k} \mathrm{~d} q \\
& =\frac{\chi_{n(i)} \chi_{n(j)}}{2 \sqrt{\zeta}} \sum_{k=0}^{d} a_{k}^{i, j} \Gamma(k+1 / 2) .
\end{aligned}
$$

$$
h_{i}(q) h_{j}(q)=\frac{\chi_{n(i)} \chi_{n(j)}}{\zeta} \exp \left(-\frac{q^{2}}{\zeta}\right) T_{i, j}\left(\frac{q^{2}}{\zeta}\right)
$$
(c)

Caruyer, E., Lenglet, C., Sapiro, G., Deriche, R., 2011b. Incremental gradient table for multiple q-shells diffusion mri, in: HBM 17th Annual Meeting, Québec, Canada.

Chan, T., Shen, J., 2005. Image Processing and Analysis - Variational, PDE, wavelet, and stochastic methods. SIAM, Philadelphia.

Cheng, J., Ghosh, A., Deriche, R., Jiang, T., 2010a. Model-free, regularized, fast, and robust analytical orientation distribution function estimation, in: Medical Image Computing and Computer-Assisted Intervention - MICCAI, Springer. pp. 648-656.

Cheng, J., Ghosh, A., Jiang, T., Deriche, R., 2010b. Model-free and analytical eap reconstruction via spherical polar fourier diffusion mri, in: Medical Image Computing and Computer-Assisted Intervention - MICCAI, pp. 590-597.

Cluskey, S., Ramsden, D.B., 2001. Mechanisms of neurodegeneration in amyotrophic lateral sclerosis. Mol Pathol 54, 386-392.

Craven, P., Wahba, G., 1985. Smoothing noisy data with spline functions. Numerische Mathematik 31, 377-403. 
Descoteaux, M., Angelino, E., Fitzgibbons, S., Deriche, R., 2006. Apparent diffusion coefficients from high angular resolution diffusion imaging: Estimation and applications. Magnetic Resonance in Medicine 56, 395-410.

Descoteaux, M., Angelino, E., Fitzgibbons, S., Deriche, R., 2007. Regularized, fast, and robust analytical q-ball imaging. Magnetic Resonance in Medicine 58, 497-510.

Descoteaux, M., Deriche, R., Bihan, D.L., Mangin, J.F., Poupon, C., 2011. Multiple q-shell diffusion propagator imaging. Medical Image Analysis 15, 603-621.

Descoteaux, M., Koay, C.G., Basser, P.J., Deriche, R., 2010. Analytical q-ball imaging with optimal regularization, in: ISMRM 18th Scientific Meeting and Exhibition.

Fillard, P., Descoteaux, M., Goh, A., Gouttard, S., Jeurissen, B., Malcolm, J., Ramirez-Manzanares, A., Reisert, M., Sakaie, K., Tensaouti, F., Yo, T., Mangin, J.F., Poupon, C., 2011. Quantitative analysis of 10 tractography algorithms on a realistic diffusion MR phantom. Neuroimage 56, 220-234.

Golub, G.H., Van Loan, C.F., 1983. Matrix computations. The John Hopkins University Press, Baltimore, Maryland.

Hansen, P.C., 2000. The 1-curve and its use in the numerical treatment of inverse problems, in: in Computational Inverse Problems in Electrocardiology, ed. P. Johnston, Advances in Computational Bioengineering, WIT Press. pp. 119-142.

Jones, D., Horsfield, M., Simmons, A., 1999. Optimal strategies for measuring diffusion in anisotropic systems by magnetic resonance imaging. Magnetic Resonance in Medicine 42, 515 - 525.

Jones, E., Oliphant, T., Peterson, P., et al., 2001. SciPy: Open source scientific tools for Python.

Koay, C.G., Özarslan, E., Basser, P.J., 2009. A signal transformational framework for breaking the noise floor and its applications in mri. Journal of Magnetic Resonance 197, 108-119.

Özarslan, E., Mareci, T.H., 2003. Generalized diffusion tensor imaging and analytical relationships between diffusion tensor imaging and high angular resolution imaging. Magnetic Resonance in Medicine 50, 955-965.

Özarslan, E., Shemesh, N., Koay, C.G., Cohen, Y., Basser, P.J., 2011. $\mathrm{Nmr}$ characterization of general compartment size distributions. New Journal of Physics 13.

Piven, J., Bailey, J., Ranson, B., Arndt, S., 1997. An mri study of the corpus callosum in autism. Am J Psychiatry 154, 1051-1056.

Poupon, C., Rieul, B., Kezele, I., Perrin, M., Poupon, F., Mangin, J.F., 2008. New diffusion phantoms dedicated to the study and validation of hardi models. Magnetic Resonance in Medicine 60, 1276-1283.

Stejskal, E., Tanner, J., 1965. Spin diffusion measurements: spin echoes in the presence of a time-dependent field gradient. Journal of Chemical Physics 42, 288-292.

Tuch, D., 2004. Q-ball imaging. Magnetic Resonance in Medicine $52,1358-1372$.

Wedeen, V., Hagmann, P., Tseng, W., Reese, T., Weisskoff, R., 2005. Mapping complex tissue architecture with diffusion spectrum magnetic resonance imaging. Magnetic Resonance in Medicine 54, 1377-1386.

You, Y.L., Kaveh, M., 2000. Fourth-order partial differential equations for noise removal. IEEE Transactions on Image Processing 9, 1723-1730. 